\title{
Migration Behaviour of Barium and Strontium through Colemanite
}

\author{
By S. Hatipoğlu ${ }^{1}$ H. Göktürk ${ }^{1}$ and H. N. Erten ${ }^{2}$ \\ 1 Department of Chemistry, Middle East Technical University, Ankara, Turkey \\ 2 Department of Chemistry, Bilkent University, Ankara, Turkey
}

(Received March 1, 1995; revised July 17, 1995)

\section{Colemanite / Sorption / Distribution ratios / Batch method / Column method}

\begin{abstract}
Summary
Column and batch experiments were performed to study the migration behaviour of $\mathrm{Ba}$ and $\mathrm{Sr}$ in colemanite matrix. ${ }^{133} \mathrm{Ba},{ }^{90} \mathrm{Sr}$ and ${ }^{3} \mathrm{H}$ were used as tracers. The retardation factors and the distribution ratios of $\mathrm{Ba}$ and $\mathrm{Sr}$ in column experiments were found to be smaller than those obtained in batch experiments. Sorption $R_{d}$ values of $\mathrm{Ba}$ were higher than those of $\mathrm{Sr}$ determined by both techniques. Sorption energies calculated from batch experiments are in good agreement with the literature values.
\end{abstract}

\section{Introduction}

The management and disposal of nuclear waste is a burden accepted along with the benefits from the use of radioactive materials. Among a number of disposal options "underground disposal" of radioactive waste seems to be the most realistic way of providing the necessary protection for humans and the environment. Colemanite $\left(\mathrm{Ca}_{2} \mathrm{~B}_{6} \mathrm{O}_{11} \cdot 5 \mathrm{H}_{2} \mathrm{O}\right)$, with its highly vitrous crystalline characteristics is a potential candidate for geological matrices considered [1], provided that it significantly sorbs the radionuclides important in waste considerations. Information on the sorption characteristics of colemanite can be obtained via batch and column experiments.

In batch experiments, the distribution ratio $R_{d}$ is obtained [2]. In column experiments, migration of a radionuclide is characterized by a retardation factor, $R_{f}$, which describes the average radionuclide velocity $\left(V_{r}\right)$ - migration rate - in the matrix with respect to the average water velocity $\left(V_{w}\right)$ in the same medium.

$$
R_{f}=\frac{V_{w}}{V_{r}}
$$

$R_{d}$ and $R_{f}$ are related by

$$
R_{f}=1+R_{d} \frac{\varrho_{s} A_{c} V_{w}}{W}
$$

where $\varrho_{s}=$ Density of the solid matrix

$A_{c}=$ Cross sectional area of the column

$W=$ Volumetric flow rate of water

Batch and column experiments provide information on the type of the sorption process, sorption ener- gies, the kinetic order of the reactions and other parameters affecting retardation.

Among the several fission products discharged into the environment, ${ }^{90} \mathrm{Sr}$ is important because of its high fission yield and long half-life. Another important safety hazard in radioactive waste considerations is $\mathrm{Ra}$. $\mathrm{Ba}$, a member of group IIA elements was chosen along with $\mathrm{Sr}$ as a suitable homolog of $\mathrm{Ra}$.

In this work the migration behaviour of $\mathrm{Ba}$ and $\mathrm{Sr}$ through colemanite has been extensively studied.

\section{Experimental}

The radionuclides ${ }^{133} \mathrm{Ba},{ }^{90} \mathrm{Sr}$ and ${ }^{3} \mathrm{H}$ obtained from the Radiochemical Center Amersham, were used as radiotracers. Colemanite was obtained from a deposit in the Emet-Kütahya region of Turkey. Fourier transform infrared and X-ray diffraction spectrometry, differential thermal gravimetric analysis, particle size fractionation and surface area measurements were carried out to elucidate the structure and properties of colemanite.

All solutions were prepared using synthetic groundwater with similar composition as the groundwaters found in Kütahya region (Table 1).

In column experiments, the retardation of radionuclides were determined by measuring the effluent activities collected from the bottom of a mini-column system $(0.32 \mathrm{~cm}$ in diameter and length of $28.7 \mathrm{~cm})$. The column was packed with a weighed amount of colemanite having $90 \mu \mathrm{m}$ average particle size. After four days of pre-equilibration with groundwater, a spike of radionuclide solution $\left(0.3 \mathrm{ml}, 5.5 \cdot 10^{4} \mathrm{~Bq}\right.$ $\left[\mathrm{Ba}^{2+}\right]=1.6 \times 10^{-8} \mathrm{mmol} / \mathrm{ml},\left[\mathrm{Sr}^{2+}\right]=5.7 \times 10^{-8}$ $\mathrm{mmol} / \mathrm{ml}$ ) was introduced from the top by injection. Then migration through colemanite was initiated with a continuous water flow through the column and fractions of effluent were collected by a fractional collector at certain time intervals. The activities of the solutions were determined by $\gamma$-rays spectrometry $\left({ }^{133} \mathrm{Ba}\right)$,

Table 1. Composition of synthetic groundwater used in the sorption experiments

\begin{tabular}{ccccccccc}
\hline \multicolumn{4}{c}{ Ion concentration (meq/L) } & & & $\mathrm{pH}$ \\
\cline { 1 - 7 } $\mathrm{Na}^{+}+\mathrm{K}^{+}$ & $\mathrm{Ca}^{2+}$ & $\mathrm{Mg}^{2+}$ & $\mathrm{CO}_{3}^{2-}$ & $\mathrm{NO}_{3}^{-}$ & $\mathrm{Cl}^{-}$ & $\mathrm{SO}_{4}^{2-}$ & \\
\cline { 1 - 7 } 0.89 & 4.70 & 3.15 & 0.17 & 3.14 & 0.84 & 0.18 & 7.80 \\
\hline
\end{tabular}




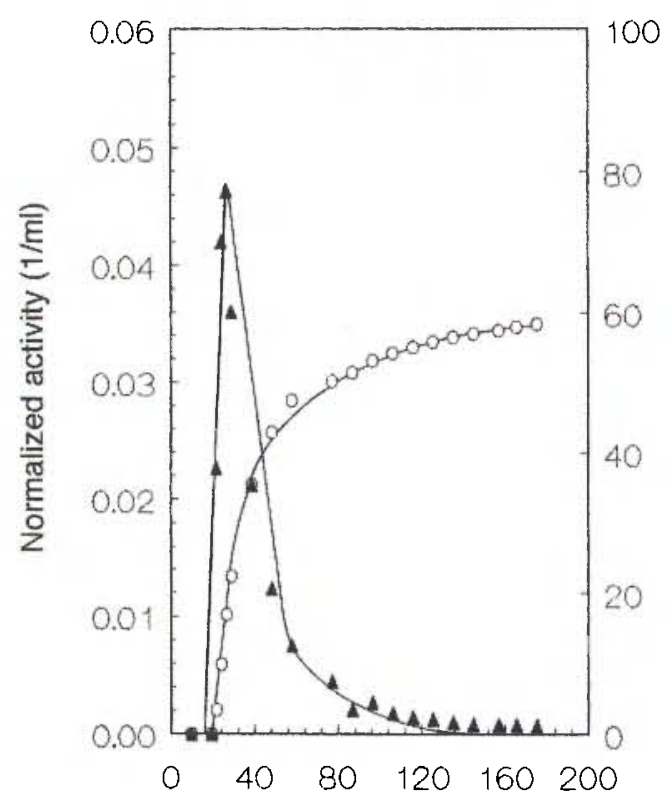

Fig. 1. A typical breakthrough curve of $\mathrm{Ba}^{2+}$-colemanite interactions. $\Delta$ : Normalized activity versus time. $\mathrm{O}$ : Cumulative eluted activity, \%.

liquid scintillation counting $\left({ }^{3} \mathrm{H}\right)$ or $\beta$-counting $\left({ }^{90} \mathrm{Sr}\right)$. Experiments were done using columns of varying length. The activities of the effluents collected were normalized as fraction of the total initial activity.

The breakthrough curve of each column experiment was obtained by plotting normalized activities per ml of effluent as a function of time. The travelling times obtained from such curves were used to calculate the transport velocity of the radionuclide in that matrix.

The average water velocity in the same matrix was obtained by performing similar experiments with tritiated water. Cumulative eluted $T$ activities were close to $100 \%$ in all cases, indicating no significant exchange of $T$.

Using the water velocity one can determine both the retardation factor, $R_{f}$, (Eq. (1)) and the distribution ratios, $R_{d}$, (Eq. (2)) in such column experiments.

In the batch technique, weighed amount of solid samples were kept in contact with known volumes of radioactive solutions of $\mathrm{Ba}$ and $\mathrm{Sr}$ for certain times. The initial concentrations ranged from $10^{-8} \mathrm{~mol} \cdot l^{-1}$ to $10^{-3} \mathrm{~mol} \cdot 1^{-1}$. After separation of the two phases by centrifugation and filtration, the change of the adsorbate concentration in the aqueous phase was measured and the distribution ratio was calculated [2].

\section{Results and discussion}

A typical experimental breakthrough curve for $\mathrm{Ba}$ migration in colemanite is shown in Fig. 1. The tailing observed indicates partially reversible adsorption [3]. The variation of the breakthrough curve with column

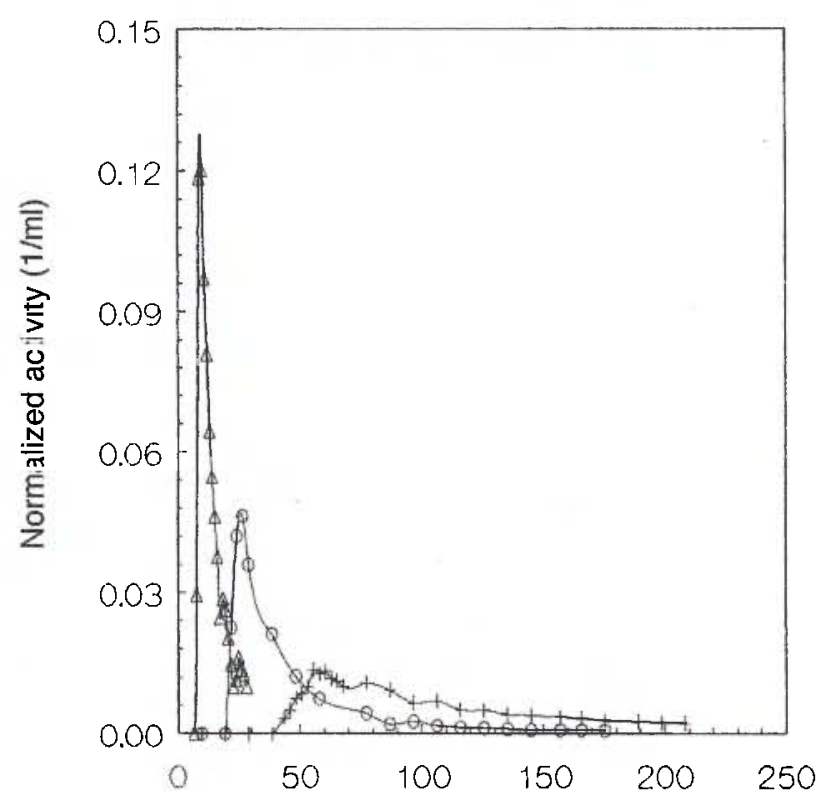

Fig. 2. Breakthrough curve of $\mathrm{Ba}^{2+}$-colemanite interactions at various column depths. $\Delta: 3.8 \mathrm{~cm} . O: 8.0 \mathrm{~cm} .+: 13.5 \mathrm{~cm}$.

length is shown in Fig. 2. The percent cumulative eluted activities are found to be 80 percent, 60 percent and 40 percent for $3.5 \mathrm{~cm}, 8.0$ and $13.5 \mathrm{~cm}$ columns, respectively. Similar behaviour was observed in $\mathrm{Sr}-$ colemanite interactions.

The velocities of $\mathrm{Ba}$ and $\mathrm{Sr}$ migration, and tritiated water were obtained by dividing the matrix depths with retention times in each column experiment. The retardation factors, $R_{f}$, were calculated accordingly. The results obtained are given in Table 2.

Assuming that a column system represents a radioactive leakage from a repository, the breakthrough curves obtained during leaching processes are treated in two different regions reflecting steady state (after the maximum activity is reached) and nonsteady state (before the maximum activity is reached) cases. The steady state equations are obtained using plots of $A_{f}$ versus time curves. Here, $A_{f}$ is the fraction of activity leached per unit time per unit volume. Then, the $\log \left(-d A_{f} / d f\right)$ versus $\log A_{f}$ (Fig. 3) plots were used to calculate the leaching rate constant, $k$, and the leaching rate order, $n$. Using these values $-\frac{d A_{f}}{d t}=k A_{i}^{n}$ type equations were obtained for $\mathrm{Ba}$ and $\mathrm{Sr}$ migration in colemanite (Table 2). Using such empirical equations, the fate of $\mathrm{Ba}$, and $\mathrm{Sr}$ ions moving through colemanite matrix, under flowing steady state conditions, can be predicted.

In the non-steady state region, assuming that $\mathrm{Ba}$ ions leach through colemanite according to first order kinetics and using the percent cumulative eluted activity information in the non-steady state region, after appropriate mathematical treatment, an empirical equation can be proposed for $\mathrm{Ba}$ ion migration as follows: 


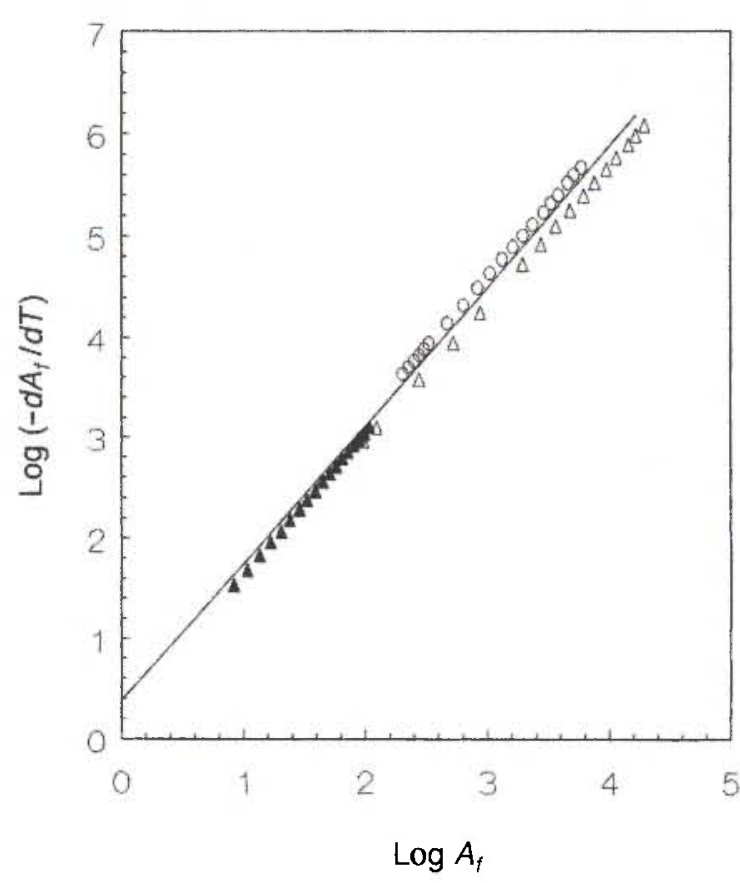

Fig. 3. Plots of $\log \left(-d A_{f} / d t\right)$ versus $\log A_{f}$ for steady state flow of $\mathrm{Ba}^{2+}$ through colemanite matrix. $\triangle$ : Matrix depth $3.5 \mathrm{~cm} . \Delta$ : Matrix depth $8.0 \mathrm{~cm}$. $\bigcirc$ : Matrix depth $13.5 \mathrm{~cm}$.

Table 2. Results of column experiments for $\mathrm{Ba}^{2+}$ and $\mathrm{Sr}^{2+}$ interactions

\begin{tabular}{|c|c|c|c|c|}
\hline & $R_{f}$ & $\begin{array}{c}R_{d} \\
(\mathrm{ml} / \mathrm{g})\end{array}$ & $-d A_{f} / d t(1)$ & $(\text { C.E.A.\% })_{\mathrm{ss}}(2)$ \\
\hline \multirow[t]{2}{*}{$\mathrm{Ba}^{2+}$} & $24 \pm 4$ & $19 \pm 3$ & $2.1 A_{f}^{1.4}$ & $\left(2.6\left(W / V_{m}\right)-0.4\right) t$ \\
\hline & & & & $1-\exp (-0.05(\mathrm{~W} / \mathrm{Vm} / \mathrm{t}))$ \\
\hline $\mathrm{Sr}^{2+}$ & $7 \pm 2$ & $5 \pm 2$ & $10 A_{f}^{1.2}$ & - \\
\hline
\end{tabular}

(1) Change of normalized activity flux of outflowing solution

(2) \% cumulative eluted activity at steady state for $0<t<$ $22\left(V_{m} / W\right)$.

$$
(\text { C.E.A.\% })_{\mathrm{ss}}=\frac{\left[2.6\left(W / V_{m}\right)-0.4\right] t}{1-\exp \left(-0.05\left(W / V_{m}\right) t\right)}
$$

where

(C.E.A.\%) ss $_{\text {: }}$ Percent cumulative eluted activity at steady state

$W / V_{m}$ : Volumetric Flowrate/Matrix Volume.

This equation is valid within $0<t<22\left(\frac{V_{m}}{W}\right)$ limits (the non-steady state region) and may be useful in predicting the maximum leachable activity of the $\mathrm{Ba}$ ion in colemanite environment.

The effect of presence of clay on the sorption characteristics of colemanite was also investigated using $75 \%$ colemanite $+25 \%$ kaolinite clay mixtures (w/w). It was observed that $R_{d}$ values increased in the presence of kaolinite from 19 to $55 \mathrm{ml} / \mathrm{g}$ in Ba sorption and from 5 to $9 \mathrm{ml} / \mathrm{g}$ in $\mathrm{Sr}$ sorption.

In batch experiments, kinetic studies indicated that the distribution ratio $R_{d}$ reached saturation in about

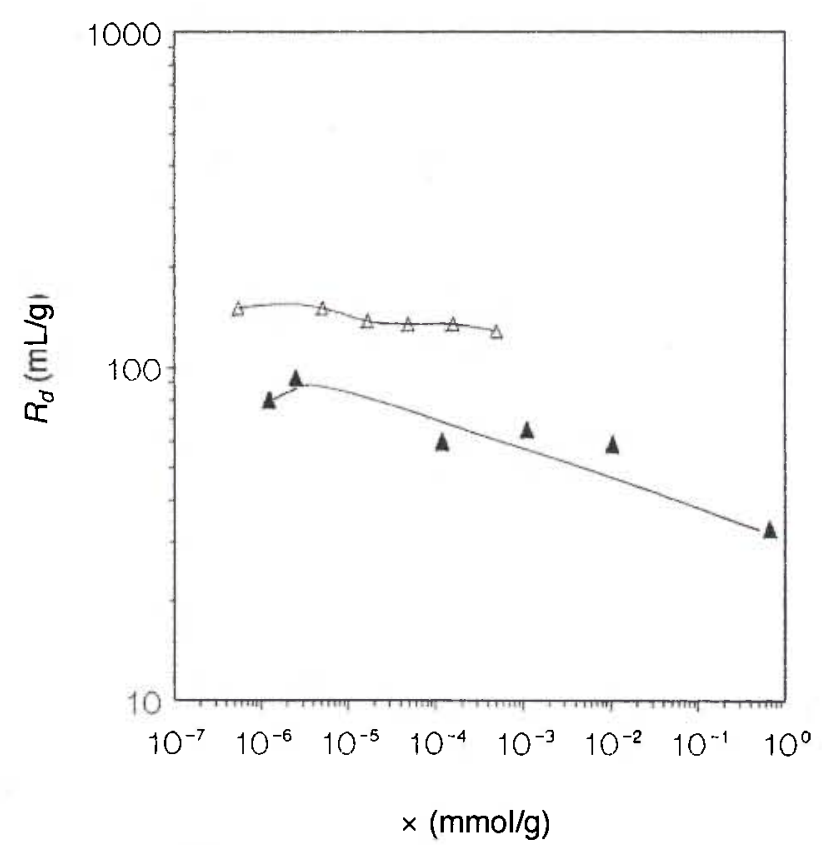

Fig. 4. Change of $R_{d}$ values as a function of cation loading in colemanite. $\triangle: \mathrm{Ba}^{2+} . \Delta: \mathrm{Sr}^{2+}$.

seven days in Ba-colemanite and in about two days in Sr-colemanite interactions.

The effect of changes in initial cation concentrations on $R_{d}$ were investigated in the $10^{-8} \mathrm{M}-$ $10^{-5} \mathrm{M}$ interval for $\mathrm{Ba}^{2+}$ and in $10^{-8} \mathrm{M}-10^{-2} \mathrm{M}$ interval for $\mathrm{Sr}^{2+}$. The resulting loading curves are given in Fig. 4. In the case of $\mathrm{Ba}$ it is apparent that the $R_{d}$ values are not a function of initial cation concentration. This suggests that probably one type of sorption site is dominating, with an average $R_{d}$ value of $140 \mathrm{ml} / \mathrm{g}$.

In $\mathrm{Sr}$ sorption, the $R_{d}$ values were found to vary slightly with the cation loading up to about $10^{-3} \mathrm{M}$. At higher concentrations, an apparent decrease in $R_{d}$ values may probably mean that energetically less favorable sites become involved in the sorption process.

The experimental data obtained in batch experiments were fitted to various sorption isotherms and are found to be well described by Freundlich [4] and Dubinin-Radushkevich [5] type isotherms. The resulting parameters obtained are given in Table 3 . Using these parameters, the corresponding Freundlich empirical isotherm for Ba-colemanite interaction can be expressed as

$$
R_{d}=95[\mathrm{Ba}]^{-002} \text {. }
$$

Here $[\mathrm{Ba}$ ] is the concentration of $\mathrm{Ba}$ in the solution following sorption. The corresponding expression for Sr-colemanite interactions is

$$
R_{d}=30[\mathrm{Sr}]^{-0.06} \text {. }
$$

Using the Dubinin-Radushkevich type isotherms similar empirical relations can be expressed:

$$
R_{d}=6.5 \times 10^{-5}[\mathrm{Ba}]^{-1} e^{\left[-5.2 \times 10^{-3}\left(R \operatorname{Tin}\left(1+[\mathrm{Ba}]^{-1}\right)\right)^{2}\right]}
$$


Table 3. Various parameters obtained in isotherm fittings

\begin{tabular}{lccc}
\hline Isotherm model & Parameter & $\mathrm{Ba}^{2+}$ & $\mathrm{Sr}^{2+}$ \\
\hline Freundlich & $k$ & 95 & 30 \\
& $N$ & 0.98 & 0.94 \\
Dubinin- & $X_{m}(\mathrm{~mol} / \mathrm{g})$ & $6.5 \times 10^{-5}$ & $3.19 \times 10^{-4}$ \\
Radushkevich & $K\left(\mathrm{~mol}^{2} / \mathrm{kJ}^{2}\right)$ & $5.19 \times 10^{-3}$ & $6.52 \times 10^{-3}$ \\
& $E(\mathrm{~kJ} / \mathrm{mol})$ & 9.8 & 8.8 \\
\hline
\end{tabular}

and

$$
R_{d}=3.19 \times 10^{-4}[\mathrm{Sr}]^{-1} e^{\left[-6.52 \times 10^{-3}(R T \ln (1+[\mathrm{Sr}]))^{2}\right]}
$$

for $\mathrm{Ba}$ and $\mathrm{Sr}$ sorption on colemanite, respectively.

The sorption energies (the free energy change when one mole of ions is transferred to the surface of the solid from infinity in solution), related to the Dubinin-Radushkevich isotherm constant $K$ (Table 3) by $E=(2 \mathrm{~K})^{-1 / 2}$ were found to be $9.8 \mathrm{~kJ} / \mathrm{mol}$ for $\mathrm{Ba}$ and $8.8 \mathrm{~kJ} / \mathrm{mol}$ for Sr-colemanite sorption. These are in good agreement with the literature values of $8-16 \mathrm{~kJ} /$ mol for ion exchange type interactions [6-8].

To conclude, the following statement can be made: From the waste management point of view, it seems that colemanite is a suitable host matrix both for radioactive $\mathrm{Sr}$ and $\mathrm{Ra}$ storage and/or disposal.

\section{Acknowledgements}

Financial supports of METU through AFP 88-01-0302 and AFP 90-01-03-05 are gratefully acknowledged.

\section{References}

1. Simon and Schuster's Rocks and Minerals, Simon and Schuster Inc. New York (1990).

2. Erten, H. N., Aksoyoğlu, Ş., Göktürk, H.: Sci. Total. Environ. 69, 269 (1988).

3. Vine, E. N., Bayhuest, B. P., Daniels, W. R., Devilliers, S. J., Erdal, B. R., Lawrance, F. O., Wolfsberg, K.: Los Alamos Scientific Laboratory Report, Los Alamos NM 87545 (1980).

4. Freundlich, H.: Colloid and Capillary Chemistry, Methuen, London (1926).

5. Dubinin, M. M., Radushkevich, L. V.: Proc. Acad. Sci. Phys. Chem. Sec., USSR (1947) 331.

6. Cerofolini, G. F.: Surf. Sci. 24, 391 (1971).

7. Hobson, J. P.: J. Phys. Chem. 81, 2720 (1969).

8. Aksoyoğlu, Ş.: J. Radioanal. Nucl. Chem. 134, 393 (1989). 